\title{
Application Analysis of Structural Vibration Control in Aseismic and Wind Resistant Bridge Design
}

\author{
Chen Xin ${ }^{1}$ \\ ${ }^{1}$ Hunan City University, Yiyang, Hunan, 413000
}

Keywords: structural vibration control; bridge design; seismic and wind resistance; ductility design

\begin{abstract}
The designer attaches great importance to seismic and wind resistance functions and two intact and independent systems in the design of bridge engineering. It is the most classic application of structural vibration control in two fields. Based on this, this paper takes the structural vibration control as the research object, and analyzes the harm and reason analysis on the vibration of the bridge caused by the earthquake and wind load, and elaborates the measures to reduce the vibration of the bridge from the aspects of anti-collision, vibration isolation and ductility design, from the structural measures, pneumatic measures and mechanical measures in the application of wind resistance and other aspects of the wind resistance measures bridge to avoid risks, protect personal safety and improve the quality of the project.
\end{abstract}

\section{Introduction}

According to relevant statistics, earthquake force and wind load are the most important natural external forces in bridge structures, posing great hidden dangers to the structure and safety of bridges. The reasons for this are mainly due to the lack of reasonable seismic and disaster-resistant design method. In recent thirty years, due to many earthquakes caused by the serious damage to the bridge structure, causing economic losses and casualties is very heavy, so according to the specific analysis of hazards, engineers design from the bridge structure vibration control, improve design measures for Bridge earthquake and wind control provide the theoretical basis [1].

\section{Hazards and Causes of Earthquake and Wind Load to the Bridge}

\subsection{The harm caused by earthquake to the bridge and its causes}

The hazards and causes of the earthquake to the bridge are as follows:

(1) There is not much damage to the superstructure and damage to the superstructure of the bridge. In the event of an earthquake, it is mainly caused by the local bending of the steel structure. The performance of the earthquake occurred, the superstructure of the bridge longitudinal displacement, lateral displacement, sometimes distorted displacement. Under normal circumstances, the easiest place to shift the earthquake damage is the bridge set expansion joints. If the superstructure of the bridge is displaced beyond the piers and platforms, there will be more serious damage to the girders.(2) from the destruction of the support, designers often do not consider the design of the support sufficient, in the connection and support structures such as the time did not take appropriate measures, and sometimes the support form and material does not meet the standards, The destruction of the bearing will directly lead to the change of the transmission of force, which will affect the rest of the bridge and further aggravate the damage caused by the earthquake. (3)The damage to the substructure and the foundation, the damage to the substructure, that is, the abutment and foundation of the bridge are mainly due to the greater level of seismic force caused by the instantaneous repeated vibration damage in the relatively weak section and even There is also the possibility of the collapse of the bridge as a whole, which is difficult to repair and repair after the earthquake[2].

The commonly used structure of the bridge is reinforced concrete pier, the main forms of damage are bending damage, shear failure and shear failure, as shown in Table 1. 
Table 1 Reinforced concrete pier damage form

\begin{tabular}{|c|c|c|c|c|}
\hline $\begin{array}{c}\text { Serial } \\
\text { number }\end{array}$ & $\begin{array}{l}\text { destruction } \\
\text { form }\end{array}$ & $\begin{array}{l}\text { destruction } \\
\text { mechanism }\end{array}$ & $\begin{array}{r}\text { destruction } \\
\text { phenomenon }\end{array}$ & Characteristics \\
\hline 1 & $\begin{array}{l}\text { Bending } \\
\text { damage }\end{array}$ & $\mathrm{Pb}<\mathrm{Ps}$ & $\begin{array}{l}\text { Plastic hinge } \\
\text { is formed in the }\end{array}$ & $\begin{array}{l}\text { There is a large deformation } \\
\text { amplitude and horizontal bending }\end{array}$ \\
\hline 2 & $\begin{array}{l}\text { Bending } \\
\text { shear } \\
\text { failure }\end{array}$ & $\begin{array}{c}\text { Bending } \\
\text { damage, } \\
\text { Shear hinge } \\
\text { drop, shear } \\
\text { failure }\end{array}$ & $\begin{array}{l}\text { damaged section. } \\
\text { Deformability }\end{array}$ & $\begin{array}{c}\text { crack in the cross section of the pier. } \\
\text { Brittle fracture, bending crack and } \\
\text { shear crack in cross section of bridge } \\
\text { pier }\end{array}$ \\
\hline 3 & $\begin{array}{l}\text { shear } \\
\text { failure }\end{array}$ & $\mathrm{Pb}>\mathrm{Ps}$ & $\begin{array}{l}\text { The ability to } \\
\text { deform is even } \\
\text { more limited. }\end{array}$ & $\begin{array}{l}\text { Brittle fracture, internal oblique } \\
\text { shear crack in the bridge pier. }\end{array}$ \\
\hline
\end{tabular}

\subsection{Hazards and causes of wind load to the bridge}

Wind load refers to the wind force on the bridge structure, that is, the result of the interaction between the wind and the bridge structure. After research and statistics, the hazards and causes of the wind load to the bridge are as follows:1) , Which is related to the stability of the problem in the structural dynamics is similar to the distortion of the column, for example, the column will reach a critical point when there will be buckling, the structure of the bridge torsion divergence, this phenomenon by the bridge structure Deflection and aerodynamic moment, rather than the ultimate strength of the bridge structure. (2)The dynamic response of bridges is mainly flutter, chattering phenomenon, galloping phenomenon and vortex-induced vibration, taking vortex-induced vibration as an example. It is a vibration phenomenon of long-span bridges under low wind speed. There are The self-excited nature and vortex-induced vibration occur in a custom region. The maximum amplitude response depends on the initial damping of the vibration system. Even small changes in the shape of the section will directly affect the response characteristics. In addition there are wind and rain vibration, the causes and forms of the water line and the cable in the wind under the influence of the harmful effects of vibration, water line vibration into the home damping negative damping [3].

\section{Seismic Measures of Bridge Based on Structural Control Theory}

\subsection{Anti-collision}

Bridge seismic anti-collision measures are:

(1) Adjust the beam width, in order to be able to adapt to changes in temperature and the impact of concrete, bridge engineers in the design and construction will be set at the appropriate location beam joints, but also for the beam Bring convenience. After researching and experimenting, it is found that the girders on both sides of the girder seam can vibrate independently when the girder is sewn, so as to absorb most of the seismic energy and reduce the harm caused by the earthquake to a certain extent. If the beam gap is too small, it will affect the temperature stress release of the beam body structure. If it is too large, it will have an impact on the driving stability of the bridge. Therefore, when designing the bridge, we can not only consider the beam gap width, but also consider it from many angles So as to reduce the impact of the earthquake to the bridge to bring the collision damage [3].

(2) Crushing device, many designers choose to install between the crushable device, which can bear the beam with the superstructure load, if the earthquake occurred, the crushing device in the collision process because of the beam body The vibrations are crushed, providing sufficient space for movement of the two sides of the beam without causing a collision, thereby reducing the energy of the earthquake. In addition, there is a crusher device is the use of seismic energy beam collision 
will focus on the crushing device, the beam end damage effectively get the link, eliminating the need for a large maintenance costs, as long as after the earthquake on the bridge pressure Aged devices can be replaced.

(3) Hard rubber cushion, rigid rubber cushion with big rigidity is installed between beam seams, which can effectively reduce the bending moment at the bottom of pier and the collision force between two beams [4].

\subsection{Vibration reducing and ductility design}

The reduced vibration isolation design of the bridge can improve the distribution of the reduced seismic force between the substructure and the abutments, protect the piers and abutments, and also can improve the torsional balance of the bridge structure so as to reduce the seismic force. The specific operation The measures are as follows:

(1)Coordinate the relationship between the seismic action of the isolated vibration-isolated bridge at different levels and the maximum allowable deformation of the superstructure and the reduction of the inertial force;

(2)The basic cycle of post- Compared with the basic cycle without using seismic isolation technology, the control should be less than 2.0;

(3)It is suggested that the bridge designer should determine the distribution relationship between the inertial forces of the bridge superstructure and the substructure in the initial stage of design, The actual actual construction laid a solid foundation.

In addition, in order to reduce the damage caused by the earthquake to the bridge, the bridge ductility design should also be fully utilized. The specific operations are as follows: (1) The types of ductile design are selected. The types of ductile structure are mainly ductile structure, finite ductile structure and fully elastic structure Under normal circumstances, the design of ordinary bridges can be completely ductile structure for earthquake resistance, its economic benefits are the greatest, but for important bridges, the design of the proposed finite ductile structure, it has the best seismic performance, for It is likely to lead to social unrest and a key bridge to national damage. It is recommended that a fully elastic structure should be adopted to ensure the normal use of the bridge even in the event of a major earthquake. (2) Capability design, you want to ensure the normal reaction ductile structure, give full play to the ductility, it is recommended to use the capacity design method for ductile seismic design, Table 2 and conventional static strength design methods to make a comparison, ability design The method has sufficient advantages[5].

Table 2 Comparison of seismic performance

\begin{tabular}{|c|c|c|}
\hline $\begin{array}{c}\text { Structural seismic } \\
\text { performance evaluation }\end{array}$ & conventional method & Capacity Design \\
\hline $\begin{array}{l}\text { The position of the plastic } \\
\text { hinge. }\end{array}$ & Uncertainty & $\begin{array}{l}\text { Predetermined component } \\
\text { parts. }\end{array}$ \\
\hline Layout of plastic hinge. & Random & preselect \\
\hline Local ductility requirements. & hard to estimate & $\begin{array}{l}\text { It is directly related to the } \\
\text { overall ductility demand. }\end{array}$ \\
\hline $\begin{array}{l}\text { Overall seismic performance of } \\
\text { the structure. }\end{array}$ & Hard to predict & Able to predict \\
\hline $\begin{array}{c}\text { Probability of preventing } \\
\text { structural collapse. }\end{array}$ & Limited & the maximum extent \\
\hline
\end{tabular}

\subsection{Damper in the seismic application}

Damped energy dissipation device can provide additional damping by local deformation of the bridge, consume or absorb the energy generated by the earthquake, reducing the seismic response of the main structure, so as to achieve the purpose of anti-vibration. Commonly used dampers are as follows: (1) friction energy dissipation damper, the device can be damping, to provide greater additional damping, independent of temperature, easy to manufacture, simple to build, the cost is 
relatively low, mainly used in structural seismic . Friction dissipative dampers are used in some of the approach bridges of China's Sutong Bridge and the Shanghai Yangtze River Bridge. When the bridge structure is deformed, the damper slides and the frictional force is generated, so that the purpose of reducing vibration is achieved. (2)Viscous damper, the device has a high energy consumption, the performance is very stable, can be repeatedly loaded and unloaded, in our use of viscous dam bridge Chongqing goose rock, the device is located in the longitudinal tightening beams and Bridge between the expansion joints Department, with the progress of science and technology and bridge design capabilities continue to improve, Lupu Bridge, the East China Sea Bridge and Pearl Bridge, etc. are used viscous damper shock absorption [6].

\section{Bridge Wind Resistance Based on Structure Control Theory}

\subsection{Structural measures}

From the perspective of bridge deformation, to improve the stiffness of the bridge, the bridge can not be a large amplitude of deformation under the action of wind load, from the energy point of view, to increase the stiffness of the structure, to improve the natural frequency of the structure, to avoid The bridge structure of high energy input, thereby enhancing the stability of the wind. From a displacement point of view, long-term wind will bring the displacement of the bridge structure, the cumulative effect of the bridge over time will have to change, so to improve the stiffness is very important. Common control measures are as follows: 1) Auxiliary cable, also known as the secondary cable, between the stay cables with high-strength steel cord connection, effectively increasing the cable frequency and coupling effect, the international Normandy France Bridge and the Second Bridge of the Yangtze River in Wuhan are used auxiliary equipment. (2) The central buckle, which is an important wind-resistant device of the new Tkmama Bridge, uses the central buckle instead of the shortest sling in the mid-span part to improve the rigidity of the bridge and improve the performance and bending resistance of the short, middle and short slings[7].

\subsection{Pneumatic measures}

Pneumatic measures can effectively improve the air flow characteristics of the bridge structure, thereby reducing the excitation of external force, the common aerodynamic measures include the addition of sealing mouth, spoiler or deflector, thereby improving the flow of air flow; , Effectively reducing the pressure difference on the surface of the beam body; it is proposed to increase the air permeability of the railings to avoid the passivation of the section due to the excessive sidewalks and curbs; and to improve the degree of streamline of the section so that the bridge structure has good aerodynamics.

\subsection{Mechanical measures in the application of wind}

Mechanical measures Dampers in the main application of the wind-resistant bridge, viscous dampers and magneto-rheological dampers. Due to the large number of flexible components in the long-span bridges, especially the cables of cable-stayed bridges, it is easy to work under the influence of the wind The amplitude response, in severe cases will lead to galloping, wind and rain vibration, the use of viscous dampers can effectively reduce the amplitude of wind vibration and improve the stability of the wind resistance, the study shows that the viscous body temperature changes viscous damper will produce a certain Therefore, the damper should be controlled at 15 degrees Celsius during the test.[7] The magnetorheological damper can input the voltage and change the damping characteristics so as to obtain the damping. By adjusting the voltage, each cable on the bridge can have better Vibration damping effect. Magnetorheological damper in the small range of speed and displacement conditions, can produce greater damping force, the effective vibration suppression in the minimum range. China's Yueyang Dongting Lake Bridge is the use of a magnetorheological damper, the installation of the damping ratio increased by 3 to 6 times, the wind-induced vibration response has also been reduced to one-twentieth. Not only viscous dampers and magneto-rheological dampers, but also permanent magnet dampers and current-variable 
dampers will all be applied to bridges depending on the actual situation. For example, the top of the Taipei 101 building is a tuned mass damper. The Liuyang River Hongshan bridge installation is a permanent magnet damper.

\section{Conclusion}

All in all, with the development of science and technology in our country and the improvement of social bridge construction, whether earthquake or wind resistance, our engineers are able to carry out the design of the bridge with ease. The seismic measures should ensure that the bridge structure will not be damaged. Strength design, wind-proof measures to ensure that the bridge structure will not instability of vibration patterns, the focus is amplitude control. From two perspectives, to strengthen the bridge design and research, through continuous testing and comparison, effectively reducing the harm caused by earthquakes and wind loads, thereby improving China's social and economic development.

\section{References}

[1] Zhang Wei. Application of structural vibration control in seismic and wind-resistant bridge design [J]., Valley, 2011 (5): 148-148

[2] Zhou Xinxu. Application of structural vibration control in seismic and wind-resistant bridge design [J]. College Construction Theory Research: 2016.6 (8)

[3] Shi Zhili, Zhou Lizhi. Research and application of seismic design and vibration control of long-span bridges [J]. Journal \& Mechatronics Eurocards. Urban Roads Bridges and Flood Control, 2002,4 (4): 7-12

[4] Liu Chun, Liu Changhong, Song Junjie. Harm of Earthquakes to Bridges, Aseismic Measures and Structural Vibration Control [J]. Journal of North Transportation, 2011 (11): 59-61

[5] Hao Chaowei, Chen Yanjiang, He Haoxiang, Wang Li-bo, Cai Jianglong. Seismic behavior analysis of high pier structural rigid frame bridge with seismic fortune time history method [J]. Journal of Disaster Prevention and Mitigation Engineering, 2017 (2): 215-221

[6] Tan Hao, Liu Zhao. Application of power spectrum method in seismic performance evaluation of bridges [J]. World Seismic Engineering, 2009,25 (4): 174-180

[7] Journal of Applied Mathematics and Mechanics, 2017,38 (1): 90-98 Comment for this article: Feedback Author Email Title Code Content Copyright by Chinese Journal of Applied Mathematics and Mechanics

[8] Zhang Yanqing, Gong Jinxin, Zhang Qin, Han Shi. Equivalent Damping Ratio of Reinforced Concrete Cylinder and Its Application in Seismic Analysis of Bridge [J]. Civil Engineering and Environment Engineering, 2015 (5): 1-10 